\title{
Lo chido de la lengua: neologismos en la juventud chilena
}

The chido about language: neologisms in Chilean youth

Gabriel Valdés-León

Universidad Católica Silva Henríquez - Chile

Santiago, Chile

gvaldesl@ucsh.cl

\section{Francisca Ceballos}

Universidad Católica Silva Henríquez - Chile

Santiago, Chile

fceballoso@miucsh.cl

Poleth Castro

Universidad Católica Silva Henríquez - Chile

Santiago, Chile

pcastrop@miucsh.cl

Gonzalo Catalán

Universidad Católica Silva Henríquez - Chile Santiago, Chile

gcatalanc@miucsh.cl

\section{Carolina Orellana}

Universidad Católica Silva Henríquez - Chile

Santiago, Chile

corellanag@miucsh.c

\section{Camila Vásquez}

Universidad Católica Silva Henríquez - Chile

Santiago, Chile

cvasquezb@miucsh.cl

\section{RESUMEN}

El objetivo de la presente investigación fue clasificar neologismos propios del léxico juvenil utilizando la clasificación propuesta por el Observatori de Neologia de la Universitat Pompeu Fabra. Para ello, se recolectaron doscientos comentarios anónimos de jóvenes de entre 13 y 18 años desde la página web y aplicación TuSecreto.io. Se procesaron los neologismos encontrados gracias al software IRAMUTEQ, con el fin de identificarlos utilizando el criterio lexicográfico, para lo cual se tomaron como obras de referencia un diccionario general (DLE) y uno regional (DUECh). Los resultados obtenidos evidencian un predominio de préstamos, con un porcentaje de $73,23 \%$, los que provienen principalmente del inglés, japonés y de la variante mexicana del español, por ejemplo, la expresión chido. Se concluye que esto puede deberse al mayor acceso que tienen actualmente los/as jóvenes chilenos a internet, lo que posibilita la conectividad y comunicación instantánea, ya sea con otras personas o con el fin de consumir productos culturales de distintas partes del mundo.

Palabras clave: léxico juvenil; discurso juvenil; neologismos; español de Chile; préstamos lingüísticos. 
The objective of this research is to classify neologisms typical of the juvenile lexicon using the classification proposed by the Observatori de Neologia of the Pompeu Fabra University. For this, two hundred anonymous comments from teens between 13 and 18 years old were collected from the website TuSecreto.io. The neologisms found were processed thanks to the IRAMUTEQ software, in order to identify them using the lexicographic criteria, for which a general dictionary (DLE) and a regional dictionary (DUECh) were taken as reference works. The results obtained show a predominance of loans, with a percentage of $73.23 \%$, which come mainly from English, Japanese and the Mexican variant of Spanish (for instance, "chido"). It is concluded that this may be due to the increasing access that young Chileans currently have to the internet, which enables instant connectivity and communication, either with other people or in order to consume cultural products from different parts of the world.

Keywords: youth lexicon; youth discourse; neologisms; Chilean Spanish; loanwords

\section{Introducción}

Los cambios que nuestra sociedad ha experimentado a lo largo de los siglos han propiciado el surgimiento de nuevas palabras; sin embargo, estas necesidades denominativas se han acrecentado en las últimas décadas gracias a la difusión masiva de nuevas unidades en los medios de comunicación y las redes sociales. Este fenómeno ha incrementado las posibilidades de líneas investigativas en el campo de la lingüística.

Diversas investigaciones han dado cuenta de ámbitos en los cuales la utilización de neologismos es frecuente, entre ellos, los discursos deportivos (Juránková, 2012), de prensa (Díaz, 2020) y juveniles (Barrera, 2020). El presente estudio se hace cargo de este último, pues se propone como objetivo identificar los neologismos propios del léxico juvenil presentes en un corpus de comentarios tomados de internet utilizando la clasificación propuesta por el Observatori de Neologia de la Universitat Pompeu Fabra.

En este documento presentamos el resultado del trabajo de búsqueda, selección, compilación y categorización de los neologismos obtenidos directamente de testimonios de jóvenes chilenos de entre 13 y 18 años, a partir de un corpus que recopiló doscientos comentarios desde la aplicación móvil llamada TuSecreto.io, aplicación en la que los/as jóvenes pueden contar anécdotas o realizar confesiones de variados temas, publicando solo su género, edad y nacionalidad. La elección de esta plataforma se debe al gran recibimiento y demanda que tiene por el público juvenil, lo que permite obtener escritos reales, anónimos y actualizados en cuanto al uso de la lengua por parte de los jóvenes.

Con la finalidad de contextualizar este trabajo, se exponen a continuación dos apartados que dan cuenta de las bases teóricas sobre las cuales se sustenta este estudio: neología léxica y léxico juvenil. El primero se encarga de las distinciones fundamentales entre neología, neologismos y su clasificación; el segundo proporciona la información necesaria para definir a la población juvenil dentro de ciertos rangos etarios e identitarios, así como también nos acerca al lenguaje utilizado por estos mismos en el internet o “ciberdiscurso” según Palazzo, (2009, p. 6). 


\subsection{Neología léxica}

Uno de los temas principales que aborda este trabajo corresponde a la neología léxica, pues los/as hablantes de cada lengua son quienes entregan movilidad a la misma, permitiendo que esta se transforme y renueve una y otra vez. Así, los estudios en este campo permiten examinar el comportamiento de los/as hablantes en cuanto a su lengua y el cómo "utilizan los mismos patrones y recursos que describen las palabras ya consolidadas en la lengua" (Cabré et al., 2002, p.161).

En primer lugar, Díaz define neología como el "proceso de producción de nuevas unidades léxicas” (2007, p. 2). Peña (2020), por su parte, se adhiere a la misma concepción que Díaz, no obstante, este autor añade un punto importante respecto a los campos lingüísticos en los que esta interviene:

La neología trabaja en los ámbitos lingüísticos del léxico (vocablo), la semántica (acepción) y la sintaxis (giro), dejando de lado la fonética y la morfología. Sin embargo, el DLE ofrece esta definición de neología "proceso de formación de neologismos”, es decir, la morfología no queda excluida del ámbito de los neologismos. [...] Así, nos encontramos que el único ámbito lingüístico que quedaría fuera del campo neológico sería la fonética (2007, p. 2).

En segundo lugar, Díaz (2007) y Valdés-León (2020) entienden los neologismos como el producto o resultado del proceso de formación de palabras, es decir, de la neología. Así también lo exponen Prat y Sierra (2011), quienes especifican que "las palabras de reciente entrada por distintas vías y aún no integradas en el léxico considerado propio son las que deben ser identificables como neologismos" (p. 145). Por su parte, Sablayrolles, propone una definición que considera tres parámetros:

...el nivel en el que se coloca el lexicólogo y los objetos que persigue constituyen un primer parámetro. Se articula ahí un segundo parámetro que tiene que ver con el problema de la difusión, que condiciona en parte el sentimiento neológico. De estos dos parámetros depende parcialmente un tercero relativo a un problema de frontera entre sintaxis y léxico (2009, p. 103).

En tercer lugar, refiriéndonos ahora a posibles clasificaciones de ambos conceptos, podemos encontrar dos tipos de neología, según Díaz (2007, p. 1):

1. Neología formal, ordinaria, de forma o de forma y sentido: responde a la creación de unidades léxicas con significantes nuevos o con significantes y significados inéditos.

2. Neología semántica o neología de sentido: esta corresponde a nuevos significados o sentidos a significantes ya existentes en la lengua.

La anterior clasificación se apoya en la propuesta de Cabré (2006), la que representa una muestra del trabajo presentado por el Observatori de Neologia (2004), organismo del cual es fundadora (Imagen 1): 
Imagen 1. Clasificación de neologismos

$\begin{array}{ll}\text { 1. Neologismos de forma } & \text { (F) } \\ \text { sufijación } & \text { FSUF } \\ \text { prefijación } & \text { FPRE } \\ \text { interferencias entre sufijación y prefijación } & \text { FPRSU } \\ \text { composición } & \text { FCOM } \\ \text { composición culta } & \text { FCULT } \\ \text { lexicalización } & \text { FLEX } \\ \text { conversión sintáctica } & \text { FCONV } \\ \text { sintagmación } & \text { FSINT } \\ \text { siglación } & \text { FTSIG } \\ \text { acronimia } & \text { FTACR } \\ \text { abreviación } & \text { FTABR } \\ \text { variación } & \text { FVAR } \\ \text { 2. Neologismos sintácticos } & \text { SINT } \\ \text { 3. Neologismos semánticos } & \text { S } \\ \text { 4. Préstamos M/AM (préstamo y préstamo adaptado, respectivamente) } \\ \text { 5. Otros }\end{array}$

Número de ocurrencias: 7021

Número de formas: 1333

Número de hápax: 800 (11.39\% de ocurrencias $60.02 \%$ de formas)

Media de ocurrencias por texto: 35.10

Fuente: Cabré (2006, p. 231).

La clasificación propuesta por Cabré es una de la más utilizadas en cuanto a estudios recientes de neología y neologismos, gracias a los nodos que utilizan la metodología y las herramientas del OBNEO (p.e., la red Antenas neológicas). Trabajos como los de Fuentes et al., (2009); Arrieta de Meza et al., (2009) y Cañete et al., (2018), junto al que aquí presentamos, dan cuenta de la aceptación que esta propuesta tiene en el ámbito de los estudios neológicos.

\subsection{Léxico juvenil}

Tal como se especificó en el apartado anterior, los/as hablantes tienden a vitalizar y enriquecer la lengua a través de la creación de neologismos. Así lo afirman Gerding et al., (2018, citando a Guerrero, 2006), quienes señalan que "si se toman en cuenta las recomendaciones, no habría que temer al préstamo ni al neologismo en general, ya que su incorporación supondría un enriquecimiento de la lengua." (p. 52).

La creación de neologismos se evidencia con fuerza en los/as jóvenes, quienes transitan por una etapa en la que se caracterizan por cuestionar o reformular cuestiones sociales, culturales, etc., previamente establecidas, las cuales ya no les parecen o consideran obsoletas:

El lenguaje juvenil se quiere diferenciar de este lenguaje estándar. Para esto los jóvenes luchan contra los tres aspectos: la norma escolar, el estilo culto y la cultura de los adultos. Sobre todo, en el caló o argot encuentran un instrumento opositor ya existente, lo que explica la existencia de muchos "préstamos" del caló en el lenguaje juvenil. (...) Pero también se explican otras características. La oposición (parcial) contra la cultura de los adultos tiene que ver con la ya citada velocidad de cambio, la que resulta de una actitud contradictoria en la valoración del lenguaje juvenil por los adultos (Zimmermann, 1996, p. 483).

Así, los/as jóvenes se abren camino en la sociedad, buscando conformar su propia identidad, tanto personal como grupal, diferente e independiente de la de sus padres, madres o adultos de su entorno (Ives, 2014). Se considera que la juventud, como grupo social, va de la mano con la idea de cambio y, dentro de este tópico de cambio, encontramos como característica relevante 
"la velocidad con que se producen determinadas modificaciones en el 'mundo conocido"” (Méndez y Rodríguez, 2011, p. 11). Esta velocidad y dinámica de "movimiento permanente" está presente también en el lenguaje; esto es lo que posibilita la creación de neologismos por parte de los/as jóvenes y la comprensión del léxico juvenil como un intento, consciente o inconsciente, de renovar el lenguaje (Méndez y Rodríguez, 2011).

Ahora bien, el rango etario que representa la juventud resulta diferente, ya que "ni siquiera en éste existe un acuerdo académico o institucional, entre distintos países, en cuanto al rango de edad a considerar como juventud. Tampoco existe acuerdo en cuanto a las justificaciones de los rangos de edad elegidos" (Taguenca, 2009, p. 165). No obstante, la Unesco ha intentado establecer un rango aproximado, definiendo la juventud entre los 15 y los 24 años (Taguenca, 2009). Para efectos de nuestra investigación, consideraremos que el discurso del cual forman parte los estudiantes chilenos de entre 13 y 18 años, cuyos comentarios conforman el corpus, presentan rasgos propios del discurso juvenil (Palazzo, 2010). Este rango etario se justifica porque corresponden a la edad estándar de los niveles educativos de enseñanza secundaria en Chile.

Actualmente, son los jóvenes quienes poseen una mayor presencia en internet (Peñafiel-Saiz et al., 2017) y, por tanto, “el lenguaje juvenil está presente en varios casos, como son: las redes sociales y los medios de comunicación, en sí está vigente en todo aquello que tiene que ver con los medios informativos" (Gallegos, 2020, p. 10).

De acuerdo con lo anterior, se debe considerar que nos referimos al lenguaje juvenil en internet como una realización particular de la lengua utilizada por jóvenes - entendiendo juventud como 'edad social' (o sea, vinculada al rol social de las personas) (Palazzo, 2009) - , y que posee características propias, como un marcado carácter oral, frecuentes transgresiones a la norma, presencia de descortesía, entre otros. Este ciberdiscurso cumple un rol principal, pues es la amalgama entre la cultura, la sociedad, la ideología y la identidad, por lo que cada joven es parte de la red y se ve representado en la lengua.

\section{Metodología}

Para realizar la investigación, se utilizó el sitio web TuSecreto.io, el cual corresponde a una página web y también a una aplicación para celular (app) en la que los usuarios pueden compartir secretos, confesiones, anécdotas y otros tipos de textos con la finalidad de comunicar e interactuar con un eventual lector/a, a través de comentarios, emojis, otras apps o redes sociales.

A partir de los datos que alberga esta plataforma, se extrajo el corpus de trabajo, pues permite filtrar el contenido por género, edad, país y año. Esto posibilitó agrupar y clasificar la información necesaria para esta investigación. De esta manera, se realizó un cuadro que ordena la información recolectada utilizando los filtros que ofrece la página web, lo que permitió extraer doscientos comentarios entre el 27 de agosto y el 08 de septiembre de 2020, escritos por jóvenes chilenos cuyo rango etario se ubica entre los 13 y los 18 años. Se seleccionó este rango etario porque interesa agrupar los neologismos que se utilizan actualmente en el léxico juvenil desarrollado en el periodo académico de la escuela secundaria en Chile, que suele corresponder con las edades señaladas. Asimismo, se extrajeron comentarios recientes con el afán de tomar una muestra del léxico juvenil utilizado en Chile en la actualidad. 
Para agrupar e identificar los neologismos del léxico juvenil presentes en los textos mencionados anteriormente, se utilizó el software IRAMUTEQ, un instrumento de clasificación lexicométrico que simplifica la búsqueda de palabras y permite realizar análisis en diferentes dimensiones. Las dimensiones de IRAMUTEQ que se tratarán en esta investigación corresponden a tipo de palabras, categoría gramatical, similitudes, etc.

Cabe señalar que, como primer paso para identificar los neologismos, seguimos el modelo propuesto por el Observatori de Neologia de la Universitat Pompeu Fabra (2004), pues, tal como se mencionó anteriormente, consiste en un modelo de clasificación ajustado a la diversidad de los datos e información. Posteriormente, una vez recopilados los datos, se enlistaron los neologismos encontrados (Cabré, 2006).

En cuanto a la recolección del corpus, reunimos 200 textos de la página web TuSecreto.io entre el 27 de agosto de 2020 y el 08 de septiembre de 2020, con el fin de clasificar aquellas palabras que resultaron novedosas. Luego, el corpus se adaptó al formato de codificación UTF-8, tal como lo exige el software IRAMUTEQ, con el fin de obtener las palabras denominadas como ' $n r$ ' (no reconocidas), según lo analizado. De acuerdo con lo anterior, el programa arrojó 1207 palabras, las cuales se encuentran clasificadas según su categoría gramatical y, además, aquellas identificadas como 'nr'. En cuanto a estas últimas, el software catalogó 439 palabras como no reconocidas, las que fueron utilizadas para el análisis. A continuación, para determinar si una palabra es un neologismo, se sigue como referencia el criterio lexicográfico (OBNEO, 2004; Fuentes et al., 2009; Valdés-León, 2020), según el cual se establece que un neologismo es todo lexema que no se recoge en el corpus de exclusión.

Como obras de referencia, fueron utilizados el Diccionario de Uso del Español de Chile (DUECh) (Academia Chilena de la Lengua, 2010) y el Diccionario de la Lengua Española (DLE) (Real Academia Española, 2016) en su versión online. Estos diccionarios servirán para identificar las palabras, establecer lo anteriormente dicho y, sobre esta base, reconocer las variantes de los vocablos que hallamos (por ejemplo, la red social Instagram se encuentra como 'IG', 'Inst4', 'Insta'). Como criterio de exclusión, consideramos no contar las diferentes formas de un mismo lema, aquellas que el software consideró “nr” por problemas de escritura ni los emoticones; en consecuencia, la selección inicial de 200 neologismos se redujo a 71. Por último, para obtener más información sobre los neologismos, se clasificaron las palabras según su categoría gramatical y el tipo de neologismo.

Sobre la base de lo anterior, los resultados obtenidos se ordenarán en dos secciones: la primera concentrará datos generales del corpus, obtenidos del software IRAMUTEQ; la segunda consiste en el análisis de los neologismos que resultan propios del lenguaje juvenil chileno.

\section{Resultados}

Para el análisis de resultados, en el primer punto se consideran datos generales, presentando información entregada por IRAMUTEQ. Aquí, se dividen aspectos propios del corpus, como número de textos, número de ocurrencias, número de formas, número de hápax y la media de ocurrencias, con el fin de entregar información general del programa. 
En el segundo punto, se expondrá la categorización de las palabras, es decir, el tipo de neologismos al que corresponde cada una, y la frecuencia de palabras, es decir, el número de veces que se presentan neologismos formales, préstamos y 'otros'.

En este mismo punto, se subdividirán dichos neologismos y préstamos en categorías gramaticales, con el fin de exponer la frecuencia en que se presentan, de mayor a menor, según corresponda. Luego, se especificarán los neologismos según su categoría y el número de veces que se muestran.

\subsection{Datos generales}

Tal como se indicó anteriormente, presentamos la información entregada por IRAMUTEQ, a partir del corpus compuesto por los 200 comentarios recopilados para el análisis. Sobre esta base, obtuvimos la siguiente información (Gráfico 1):

Gráfico 1. Resultados generales del corpus

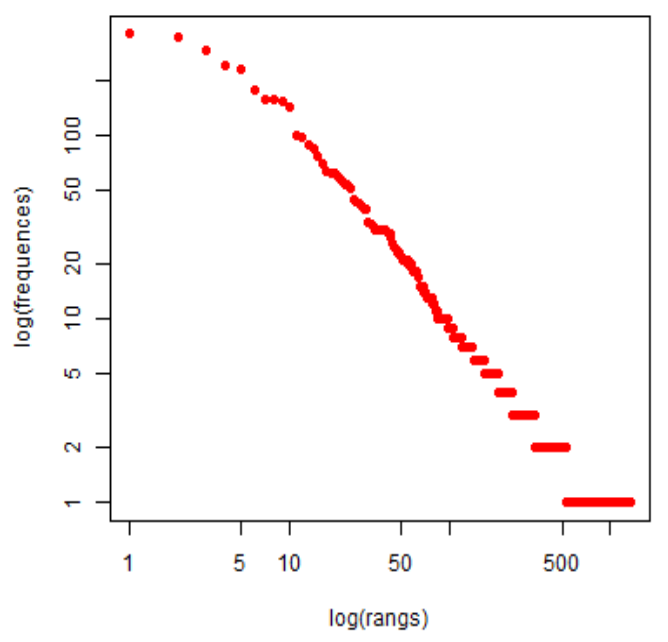

Fuente: elaboración propia

Con el fin de aclarar la información, especificaremos algunos conceptos: el número de ocurrencias responde a la cifra de unidades léxicas presentes en el corpus; el número de formas es el número de palabras presentes, sin contar las reiteraciones; por último, el número de hápax corresponde a los términos que solo se presentaron una vez en los textos.

\subsection{Análisis de neologismos}

A partir del corpus obtenido en TuSecreto.io, pudimos recolectar doscientos textos entre el 28 de agosto y el 8 de septiembre. De estos, logramos obtener 71 neologismos, los que se clasifican de la siguiente forma (Gráfico 2): 
Gráfico 2: Clasificación de neologismos presentes en el corpus

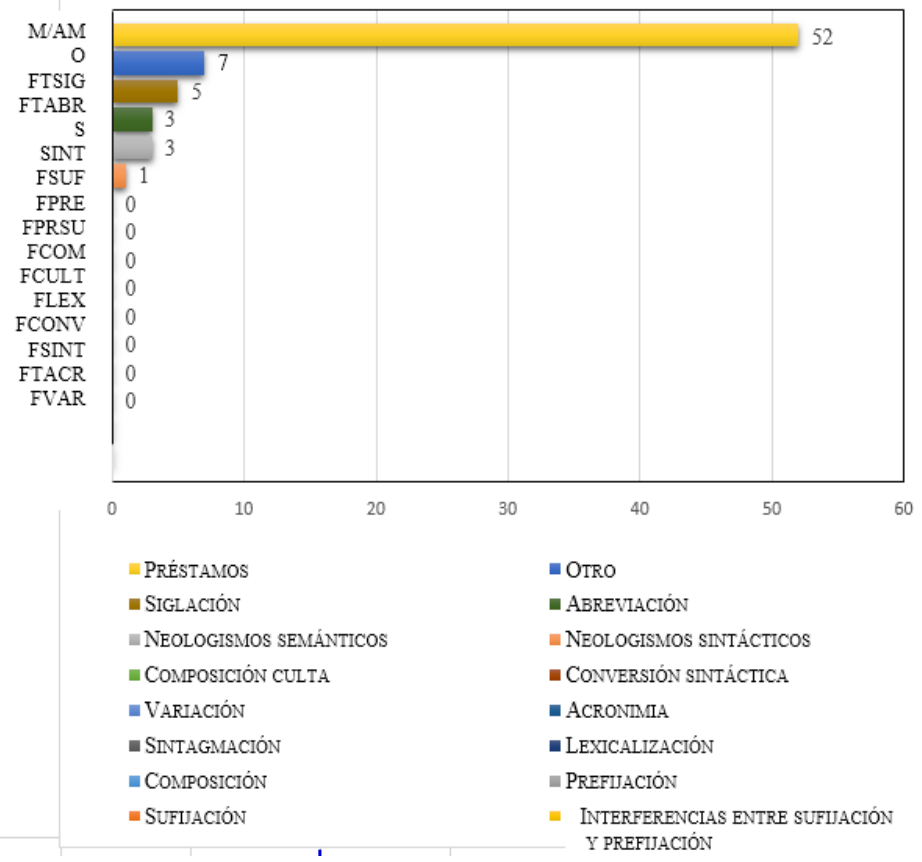

Fuente: elaboración propia

En cuanto a la frecuencia, considerando préstamos, neologismos formales y otros, se recogió la información que aparece en la Tabla 1.

Tabla 1: Frecuencia de Neologismos

\begin{tabular}{|l|c|c|}
\hline Tipos de neologismos & Valores absolutos & Porcentajes \\
\hline Préstamos & 52 & $73,23 \%$ \\
Neologismos formales & 12 & $16,9 \%$ \\
otros & 7 & $9,8 \%$ \\
\hline Total & 71 & $100 \%$ \\
\hline
\end{tabular}

Fuente: elaboración propia

\subsubsection{Tipos de neologismos}

\section{Préstamos A/AM}

Los préstamos responden a aquellas palabras que provienen de otros idiomas, los que se clasifican en adaptados y no adaptados. El primer grupo corresponde a aquellos que se han modificado según las convenciones de nuestra lengua como, por ejemplo, chipear (en este caso, se ha añadido la terminación -ear al verbo en inglés to chip). Por su parte, los no adaptados son los que se mantienen en su lengua original como hentai, like y psycho, entre otros.

Además, se encontraron algunos préstamos que tienen problemas de escritura, como son las palabras del japonés Chidori y Fujoshi (escritos erróneamente como shidori y fijoshi). Por su parte, destacan neologismos como mainkra y creizy, los que denotan una adaptación parcial a las reglas ortográficas del español de Minecraft y crazy, respectivamente. 
A partir de los resultados, son los préstamos los que poseen mayor frecuencia en el vocablo juvenil. De esta forma, en la Tabla 2, los clasificamos de acuerdo con la lengua de procedencia, su adaptación al español y su porcentaje de aparición.

Tabla 2: préstamos presentes en el corpus

\begin{tabular}{|l|c|c|c|c|}
\hline Lengua & $\begin{array}{c}\text { No adapta- } \\
\text { dos }\end{array}$ & Adaptados & $\begin{array}{c}\text { Valores abso- } \\
\text { lutos }\end{array}$ & Porcentaje \\
\hline Inglés & 38 & 3 & 41 & $78,8 \%$ \\
Japonés & 7 & 0 & 7 & $13,4 \%$ \\
Español (variante mx) & 3 & 0 & 3 & $5,7 \%$ \\
Español (variante argentina) & 1 & 0 & 1 & $1,9 \%$ \\
\hline Total & 49 & 3 & 52 & $100 \%$ \\
\hline
\end{tabular}

Fuente: elaboración propia

\section{A) Neologismos sintácticos (SINT)}

Los neologismos sintácticos corresponden a aquellos que sufren cambios en sus características gramaticales (género, número, cambio en la naturaleza verbal, entre otros) como, por ejemplo, simpean (de simpear, utilizado como verbo en el corpus, pero proveniente del adjetivo inglés $\operatorname{simp})$.

\section{B) Neologismos por abreviación (FTABR)}

Corresponden a aquellos neologismos que se forman por abreviación de la base léxica: pls (please), app (application) o wsp (WhatsApp). En cuanto a las variantes halladas, se puede observar que el lexema que presenta más variantes es instagram: inst4, instgram, îns5a, înst4 e ig.

C) Neologismos por siglación (FTSIG)

Corresponde a aquellas palabras que se forman a partir de algunos elementos que pertenecen al lexema inicial como, por ejemplo, $2 d$ (bidimensional), wtf (what the fuck), ps4 (PlayStation 4), entre otras.

\section{D) Neologismos semánticos (S)}

Estos términos se crean a partir de una base léxica existente, pero que cambia su significado. A modo de ejemplo: panas (referido a amigos/as), fleta (sinónimo de lesbiana), cuea (referido a suerte).

\section{E) $\operatorname{Otros}(\mathrm{O})$}

En este grupo, se ubican las palabras que resultan difíciles de clasificar dentro de los neologismos formales o préstamos lingüísticos. Ejemplos de estos casos son $x d$, ewe, wow, unu, etc. En el caso particular de la palabra $x d$, esta posee la variante $x d d$, lo que podría explicarse como un intento de entregar un mayor énfasis en lo emocional.

\subsubsection{Categorización gramatical}

De acuerdo con los neologismos encontrados, como se aprecia en la Tabla 3, podemos determinar que la categoría gramatical con mayor presencia es el nombre, seguido por la interjección. Las otras categorías presentes son adjetivos, verbos, pronombres y adverbios, siendo esta última la de menor uso. 
Tabla 3: Categoría gramatical de los neologismos

\begin{tabular}{|l|c|c|}
\hline Categoría gramatical & Valor absoluto & Porcentaje \\
\hline Nombre & 44 & $61,97 \%$ \\
Interjección & 10 & $14,08 \%$ \\
Adjetivo & 8 & $9,40 \%$ \\
Verbo & 5 & $7,04 \%$ \\
Pronombre & 3 & $4,22 \%$ \\
Adverbio & 1 & $1.40 \%$ \\
\hline Total & 71 & $98,11 \%$ \\
\hline
\end{tabular}

Fuente: elaboración propia

\section{Discusión}

A partir de la información presentada en la Tabla 1, podemos afirmar que el neologismo que se utiliza con mayor frecuencia es el préstamo, con un porcentaje de $73,23 \%$. Al respecto, la investigación de Fuentes et al., (2009), que analiza neologismos presentes en prensa chilena entre los años 2003 y 2006, da cuenta de resultados similares: el préstamo como el tipo de neologismo con mayor productividad y, como era de esperar, el nombre como la categoría gramatical predominante. Si bien los resultados no son equiparables, dado el enfoque de cada investigación, estos invitan a reflexionar en torno a los mecanismos a través de los cuales se incorporan los neologismos en las distintas esferas sociales de una lengua.

En contraste con lo anterior, el estudio de Torrego González (2011), enfocado en el léxico juvenil español, enfatiza en que los jóvenes que ocupan la red social Tuenti suelen usar siglación o abreviación de palabras, haciendo del lenguaje una especie de código secreto que solo puede ser entendido por los/as internautas de dicha aplicación. En cuanto a los préstamos, se señala que se ocupan de forma secundaria, como un “(...) factor de naturaleza estética” (p. 17). En otras palabras, los/as jóvenes utilizan ciertos anglicismos para llamar la atención de los/as demás usuarios/as. En la misma línea, la investigación realizada por Gallego (2017) sobre neologismos utilizados en el contexto del acoso escolar en España identifica que los/as estudiantes suelen ocupar palabras propias del idioma español, dejando en segundo lugar anglicismos tales como troll o hater.

En el ámbito americano, los hallazgos parecen diferir a los que plantean Torrego González (2011) y Gallego (2017). Por ejemplo, en cuanto a neologismos juveniles en América, Abril y Moscoso (2018) entrevistan a 470 estudiantes universitarios en relación con el léxico empleado en redes sociales y concluyen, al igual que la presente investigación, que los préstamos del inglés tienen un papel preponderante. Similares resultados encuentran Henao-Cardona y Taramuel (2018), quienes abordan la influencia que las TIC han tenido en el lenguaje juvenil, con énfasis en Colombia, y destacan también la alta presencia de anglicismos en este rango etario.

Evidentemente escapa del alcance de nuestro estudio indagar en la valoración que el español americano concede a los préstamos (sobre todo del inglés) en oposición a la que se le otorga en el español peninsular; no obstante, trabajos como el de Van Hooft (2006) y el de Gerding, Cañete y Adam (2018) nos llevan a pensar que dicho factor podría explicar tales diferencias. 


\section{Conclusiones}

En la presente investigación, se identificaron los neologismos que se encuentran en el léxico juvenil chileno en doscientos textos extraídos de la página web TuSecreto.io, en los cuales se hallaron 71 neologismos, los que se clasificaron según el modelo propuesto por el Observatori de Neologia de la Universitat Pompeu Fabra.

De acuerdo con los resultados de esta clasificación, se puede concluir que los neologismos más utilizados son los préstamos, que provienen mayoritariamente del inglés, del japonés y, en menor medida, del español mexicano. Esto probablemente se debe al acceso que tienen los/as jóvenes al internet, herramienta que posibilita la conectividad y comunicación instantánea entre personas en distintas plataformas que se utilizan como redes sociales y, además, favorece el consumo de productos audiovisuales de diversas latitudes.

En lo que concierne a los hallazgos, podemos constatar variantes como la combinación alfanumérica de algunas palabras escritas en redes sociales, como por ejemplo 19 , inst4, ins5a, etc. Asimismo, encontramos la simplificación de palabras por parte de los/as jóvenes, lo que responde a la instantaneidad y la rapidez propia del internet. Asimismo, destaca la escritura de palabras de acuerdo con su composición fonética o fonológica, lo que implica la transgresión de ciertas reglas ortográficas del español.

Ahora bien, una posible proyección de lo revisado en la investigación es el análisis discursivo de los neologismos, considerando el contexto en el que se emplea cada pieza léxica, así como también los factores sociales y culturales que podrían tener implicancia en su utilización.

Evidentemente, los resultados de esta investigación no son generalizables; no obstante, representan una contribución a los estudios del léxico en tanto da cuenta de algunas características de los neologismos utilizados por la juventud chilena, características que se ven reflejadas en otros ámbitos de la lengua chilena (por ejemplo, el discurso periodístico) y que aparecen, con menor preponderancia, en jóvenes de otras latitudes como el caso de Perú y España.

\section{Referencias}

Abril Yaco, A. G., \& Moscoso Allain, J. E. (2019). Vocabulario empleado en redes sociales por los universitarios: tipología y recursos de los neologismos (Tesis de grado). Universidad Nacional de San Agustín de Arequipa, Arequipa.

Academia Chilena de la Lengua. 2010. Diccionario de uso del español de Chile. MN Editorial Ltda

Arrieta de Meza, B., Meza Cepeda, R., \& Batista, J. (2009). Clasificación de neologismos usados en el lenguaje académico por docentes universitarios venezolanos. Opción, 25(59), 53-64. https:// produccioncientificaluz.org/index.php/opcion/article/view/6471

Barrera, O. (2020). Influencia de los neologismos en la adquisición del léxico de los estudiantes de 8vo de básica y 1ero de bachillerato paralelo "A" del "Colegio Eduardo Salazar Gómez", en el año lectivo 2019 - 2020 (Tesis de grado) Universidad Central de Ecuador.

Cabré, M. T., Bayà, M., Bernal, E., Freixá, J., Solé, E., \& Vallès, T. (2002). Evaluación de la vitalidad de una lengua a través de la neología: a propósito de la neología espontánea y de la neología planificada. En M. T. Cabré, J. Freixa y E. Solé (eds.), Lèxic i neologia, (pp. 159-201). Universitat Pompeu Fabra. 
Cabré, M. T. (2006). La clasificación de neologismos: una tarea compleja. Alfa, 50(2) 229-250. https:// periodicos.fclar.unesp.br/alfa/article/view/1421

Cañete, P., Fernández-Silva, S., \& Villena, B. (2018). Estudio de los neologismos terminológicos difundidos en el diario El País y su inclusión en el diccionario. B. CLAC 80, 135-158. https://doi.org/10.5209/ clac.66604

Díaz, M. (2007). Aproximación lingüística a la neología léxica. En J. C. Martín Camacho y M. I. Rodríguez Ponce (eds.), Morfología: Investigación, docencia, aplicaciones. (pp. 33-55). Universidad de Extremadura.

Díaz, M. (2020). Neología y prensa escrita: claves de unas interrelaciones necesarias. Tonos Digital, (38), 1-38. http://www.tonosdigital.es/ojs/index.php/tonos/article/view/2399

Fuentes, M., Gerding, C., Pecchi, A. Kotz, G. y Cañete, P. (2009). Neología léxica: reflejo de la vitalidad del español de Chile. Revista de Lingüística y Aplicada, 47 (1), 103-124.

Gallego, M. (2017). La agresión verbal. Neologismos y adaptaciones léxicas en la educación secundaria. Una propuesta de web-quest. Almería: Universidad de Almería.

Gallegos J., N. (2020). La influencia de la jerga juvenil en el lenguaje de los jóvenes estudiantes (Tesis de grado). Universidad Central del Ecuador.

Gerding Salas, C., Cañete González, P., \& Adam, C. (2018). Periodistas: ¿̇usar o no usar préstamos? he ahí la cuestión. Nueva revista del Pacífico, (68), 42-61. http://www.nuevarevistadelpacifico.cl/index.php/ $\mathrm{NRP} /$ article/view/110

Henao-Cardona, E., \& Taramuel, T. (2017). Cambios en la escritura juvenil favorecidos por las TIC. Ventana Informática, (37), 121-132. https://doi.org/10.30554/ventanainform.37.2726.2017

Ives, E. (2014). La identidad del Adolescente. Cómo se construye. Revista de formación continuada de la sociedad española de medicina de la adolescencia, 2(2), 14-18.

Juránková, A. (2012). Los neologismos en artículos deportivos y políticos de El Mundo: Análisis y comparación. Universidad Masarykova. Facultad de Filología. Masarykova univerzita Filozofická fakulta

Méndez, S., \& Rodríguez, E. (2011) Consecuencias futuras del despertar de una generación de adolescentes digitales. Escenarios posibles. Revista de estudios de juventud, 92, 11-36.

Observatori de Neologia. (2004). Metodologia del trabajo en neología: criterios, materiales y procesos. Institut Universitari de Lingüística Aplicada/Uni-versitat Pompeu Fabra, Papers de l' IULA.

Palazzo, M. (2009). El ciberdiscurso juvenil: representaciones sociales del desconcierto, la censura y la aceptación. Espéculo. Revista de estudios literarios. Universidad Complutense de Madrid, 41. https://webs. ucm.es/info/especulo/numero41/ciberdis.html

Palazzo, M. (2010). Aspectos comunicativos del ciberdiscurso juvenil. Consideraciones teóricas. Revista Argentina de Estudios de Juventud, (3), 1-22. https://perio.unlp.edu.ar/ojs/index.php/revistadejuventud/ article/view/1486

Peña, E. (2020). Aproximación a los neologismos léxicos del español actual en el ámbito de las TIC (Tesis de maestría). Universitat de les Illes Balears, Palma de Mallorca.

Peñafiel-Saiz, C., Ronco-López, M., \& Echegaray-Eizaguirre, L. (2017). Jóvenes, salud e Internet. Percepción, actitud y motivaciones de los jóvenes ante la información de salud. Revista Latina De Comunicación Social, (72), 1317-1340. https://doi.org/10.4185/RLCS-2017-1221

Prat, M., \& Sierra, S. (2011). Los neologismos en la sociedad de la información: análisis de su presencia y ausencia en las fuentes lexicográficas escolares. Teoría de la Educación. Educación y Cultura en la Sociedad de la Información, 12(3), 141-164. https://doi.org/10.14201/eks.8487

Real Academia Española (2016). Diccionario de la lengua española, 23. ${ }^{a}$ ed. Espasa. Disponible en: http://dle. rae.es/

Sablayrolles,J.-F.(2009). ¿Neologismo o no? Ensayo de clarificación de algunos problemas de incorporación. Revista de Investigación Lingüística, (12), 101-122. https://revistas.um.es/ril/article/view/91271

Taguenca Belmonte, J. (2009). El concepto de juventud. Revista mexicana de sociología, 71(1), 159-19o. http:// dx.doi.org/10.22201/iis.01882503p.2009.001.17746 
Torrego González, A. (2011). Algunas observaciones acerca del léxico en la red social Tuenti. Tonos. Revista electrónica de estudios filológicos, 21, 1-16.

Valdés-León, G. (2020). Reflexionando con ‘chispeza’: ¿Y si la añadimos al diccionario? Religación. Revista de Ciencias Sociales y Humanidades 5(25), 240-247. http://dx.doi.org/10.46652/rgn.v5i25.689

Van Hooft, A. (2006). El valor añadido de los anglicismos en la publicidad española. El impacto y la valoración social de las voces inglesas en los anuncios de la revista Elle. En M. V. Calvi \& L. Chierichetti (Eds.), Nuevas tendencias en el discurso de especialidad (pp. 219-243). Lang.

Zimmermann, K. (1996). Lenguaje juvenil, comunicación entre jóvenes y oralidad. En T. Kotschi, W. Oesterreicher y K. Zimmermann (eds.), El español hablado y la cultura oral en España e Hispanoamérica. (pp. 475-514). Vervuert/ Iberoamericana.

\section{AUTORES}

Gabriel Valdés-León. Doctor en Lingüística, Universidad de Las Palmas de Gran Canaria. Académico de la Escuela de Educación en Castellano, Universidad Católica Silva Henríquez.

Francisca Ceballos. Licenciada en Educación y Profesora de Lengua y Literatura de la Universidad Católica Silva Henríquez, Chile

Poleth Castro. Licenciada en Educación y Profesora de Lengua y Literatura de la Universidad Católica Silva Henríquez, Chile

Gonzalo Catalán. Licenciado en Educación y Profesor de Lengua y Literatura de la Universidad Católica Silva Henríquez, Chile

Carolina Orellana. Licenciada en Educación y Profesora de Lengua y Literatura de la Universidad Católica Silva Henríquez, Chile

Camila Vásquez. Licenciada en Educación y Profesora de Lengua y Literatura de la Universidad Católica Silva Henríquez, Chile

\section{Conflicto de intereses}

El autor(es) informa(n) ningún conflicto de interés posible. Financiamiento

No existió asistencia financiera de partes externas al presente artículo". Agradecimientos

N/A

Nota

Aportes de los autores.

Gabriel Valdés-León: Concepción y diseño de la investigación. Discusión bibliográfica.

Francisca Ceballos: Redacción y revisión del marco teórico

Poleth Castro: Redacción y revisión del marco teórico

Gonzalo Catalán: Búsqueda y procesamiento de los datos

Carolina Orellana: Análisis e interpretación de los datos, conclusiones

Camila Vásquez: Conclusiones, selección del corpus, revisión final 